\title{
Biotransformation of 1- and 2-phenylethanol to products of high value via redox reactions
}

\author{
Agata Geą *, BeAta Szmigiel-Merena, \\ MAŁgORZATA BRZEZIŃSKA-RODAK, EWA ŻYMAŃCZYK-DUDA \\ Department of Bioorganic Chemistry, Wrocław University of Science and Technology, Wrocław, Poland
}

\begin{abstract}
The aim of this research was to conduct biotransformation of phenolic compounds, prochiral ketone acetophenone into optically pure $(R)$ - and $(S)$-1-phenylethanol and 2-phenylethanol into tyrosol and hydroxytyrosol. For acetophenone reduction reaction, the biocatalysts of choice were cyanobacteria, phototrophic microorganisms with biocatalytic potential that have not yet been fully discovered. For the hydroxylation reaction of 2-phenylethanol, instead of using vital Aspergillus niger cells, the reaction was carried out using the spores. As a result, high conversion rates were obtained (79.6\%) for the reduction reaction performed by photobiocatalyst Nodularia sphaerocarpa, although the enantioselectivity was moderate (enantiomeric excess, ee 48\%). The reaction carried out by cyanobacterium Leptolyngbya foveolarum yielded 25.7\%, but with high enantioselectivity of ( $S$ )-1-phenylethanol $95 \%$ ee was received. It has also been proven that the acetophenone reduction did not depend on the light regime. For aromatic ring hydroxylation, several modifications of the conditions of biooxidation were introduced to maximize the effectiveness; the addition of glucose into the reaction medium gave the best results. As a result, new methods of bioconversion of phenylethyl alcohol have been developed, leading to the formation of desired products: 1-phenylethanol, tyrosol, and hydroxytyrosol antioxidants, compounds of utilitarian meaning.
\end{abstract}

Key words: spores, cyanobacteria, Aspergillus niger, phenylethanol, biocatalysis, tyrosol

\section{Introduction}

Phenolic compounds are of high commercial value owing to their multiple applications. Chiral 1-phenylethanol can be obtained through biotransformation of acetophenone, a substrate which is used in food and cosmetic industries due to its rose-like flavor (Muhr et al., 2016). Optically pure $(R)$ - and $(S)$-1-phenylethanols are two of the most important chiral building blocks in pharmaceutical and cosmetic industries (Cao et al., 2012). 2-phenylethanol is an aromatic alcohol, widely occurring in nature in essential oils of many plants. It has a rose-like aroma and it is a common ingredient in flavor and fragrance industry (Stark, 2002; Schrader et al., 2004). Tyrosol and hydroxytyrosol are derivatives of hydroxylated 2-phenylethanol and they possess extraordinary antioxidant activities. These polyphenols commonly occur in olive oil, and they are usually obtained in a process which requires expensive extraction methods (Franco et al., 2014; Ting Hu et al., 2014).
Antioxidants are compounds that have the ability to neutralize free radicals - molecules or atoms with unpaired valence electrons that are highly chemically reactive toward other substances and therefore cause damage to the living cells, resulting in many diseases. Antioxidants have a positive effect in the treatment and prevention of illnesses such as cancer, cardiovascular diseases, diabetes, brain strokes, and skin diseases. They also delay the process of aging in humans. The knowledge about destructive effects of free radicals induces constant searching for new substances that are able to prevent damages or for novel modification of the known ones (Andreassi and Andreassi, 2004; Umeno et al., 2015).

Biocatalysis is an interesting alternative for organic chemistry methods. It provides simple, cost-effective, and economically justified synthesis of desired molecules. Whole cell biocatalysts are also advantageous as they have low cost and stability, as well as high regio-, 


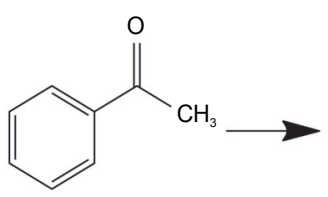

Acetophenone

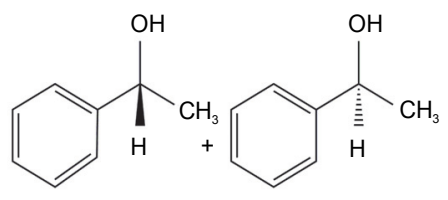

(R)-1-phenylethanol
(S)-1-phenylethanol
Fig. 1. Enantioselective biotransformation of acetophenone<smiles>OCCc1ccc(O)cc1</smiles>
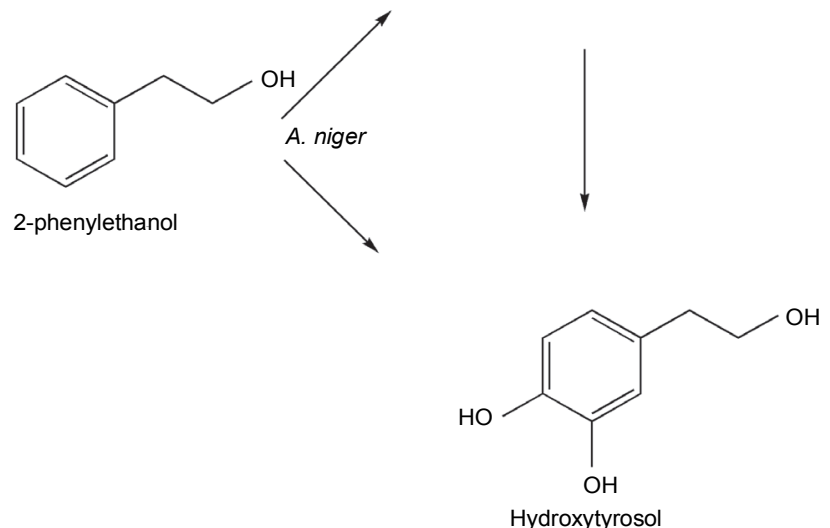

Fig. 2. Possible routes of biohydroxylation

stereo-, and enantioselectivity of the reaction. Furthermore, enzyme purification and cofactor addition can be avoided (Faber, 2000).

The aim of the study was to use microorganisms for the enantioselective synthesis of 1-phenylethanol from acetophenone (Fig. 1) and for the production of antioxidants: tyrosol [2-(4-hydroxyphenyl)ethanol] and hydroxytyrosol [2-(3,4-dihydroxyphenyl)ethanol], using 2-phenylethanol as a starting material (Fig. 2). The main idea was to elaborate a method of conversion of inexpensive starting materials into polyphenols having specific biological properties and chiral building blocks in optically pure forms.

Although the enantioselective reduction of acetophenone to the corresponding chiral alcohols has been thoroughly studied and described (Kisikuri and Andrade, 2015), cyanobacteria, which have recently gained much attention, thanks to their wide application in biotechnology (Abed et al., 2009), are new biocatalysts with scarce application in this field (Havel and Wuester-Botz, 2006). Moreover, using spores of Aspergillus niger as biocata- lysts in the biohydroxylation reaction is not a common approach. It is a relatively new method that directly uses spores of $A$. niger which are recovered from solid medium Petri dishes, without the need for inoculation in a liquid medium. Compared to vegetative cells, spores are resistant to toxic compounds, temperature, or desiccation (Wolken and Tramper, 2003). Experiments that use spores for compounds synthesis, for example, biotransformation of citral and nerol by spores of Asperigillus niger or Penicillium digitatum have already been described in the literature (Demyttenaere and Pooter, 1998; Esmaeili et al., 2011).

\section{Material and methods}

\section{Microorganisms}

Aspergillus niger (OPI) strain was obtained from Opole University (Poland). All cyanobacteria species used in the research: Arthrospira maxima (CCALA 27), Geitlerinema sp. (CCALA 138), Leptolyngbya foveolarum (CCALA 76), Nodularia sphaerocarpa (CCALA 114), Nostoc cf-muscorum (CCALA 129), and Synechococcus bigranulatus (CCALA 187), were obtained from the Culture Collection of Autotrophic Organisms (CCALA) (Institute of Botany, Academy of Sciences of the Czech Republic).

\section{Cultivation conditions}

Cyanobacteria were cultivated in $250 \mathrm{ml}$ Erlenmeyer flasks with $100 \mathrm{ml}$ of growth medium. Arthrospira maxima was grown on Spirulina medium (Aiba and Ogawa, 1977), whereas Geitlerinema sp., Leptolyngbya foveolarum, Nodularia sphaerocarpa, and Synechococcus bigranulatus were grown on BG-11 medium (Rippka et al., 1979). Nostoc cf. muscorum, as a microorganism with nitrogen fixation ability, was cultivated on BG-11 medium without sodium nitrate. The cultures were grown under sterile, stationary conditions, at $30^{\circ} \mathrm{C}$ and under continuous illumination provided by fluorescent lamps (Power-Glo, 8W, Hagen; SunGlo, 8W, Hagen; Sylvania, $8 \mathrm{~W}$, Gro-Lux).

Aspergillus nigerwas cultivated on a potato dextrose agar (PDA). The solid agar medium (diameter of Petri dishes $14 \mathrm{~cm}$ ) was inoculated with $A$. niger. Cultures were incubated at $25^{\circ} \mathrm{C}$ for 4 days. After 4 days, the surfaces of Petri dishes were covered with spores and biotransformation reactions were initiated. 


\section{Procedure of biotransformation}

\section{Acetophenone reduction}

After 3 weeks of pre-cultivation under stationary conditions and continuous illumination provided with different light sources at $30^{\circ} \mathrm{C}, 1 \mathrm{mM}$ of acetophenone $(11.7 \mu \mathrm{l})$ was added to the axenic culture of cyanobacteria. After 7 days of conversion under the same conditions, the process was completed by centrifugation $(2500 \mathrm{~g}, 20 \mathrm{~min})$. The supernatant was extracted twice with ethyl acetate $(2 \times 50 \mathrm{ml})$. Then, the organic layer was dried over anhydrous $\mathrm{MgSO}_{4}$ and evaporated under reducing pressure (Rotary evaporators IKA $\AA$ RV10 digital). The prepared samples were analyzed using gas chromatography (GC, model 7890A; Agilent Technologies Co., Ltd.), equipped with a Chiralsil DEX CB column $25 \mathrm{M} \times 0.25 \mathrm{~mm}$.

Control experiments were performed without the presence of cyanobacteria cells or without the reaction substrate.

\section{Biohydroxylation of 2-phenylethanol}

Spores of Aspergillus niger were recovered from sporulated surface cultures on a solid agar medium - they were washed with sterile water and Triton X-100 solution $(0.05 \%)$, suspended and transferred to conical flasks $(250 \mathrm{ml})$. The number of spores of Aspergillus niger obtained from sporulated Petri dishes (inoculum) was about $20000 / \mu 1$, which was measured by a total cell counter (FACSVerse flow cytometer). The biotransformation medium consisted of $20 \mathrm{ml}$ of spore suspension, $10 \mathrm{ml}$ sterile distilled water, and the following amounts of the 2-phenylethanol as a substrate: $15 \mathrm{mg}(4 \mathrm{mM})$; $30 \mathrm{mg}(8 \mathrm{mM}) ; 60 \mathrm{mg}(17 \mathrm{mM})$. The other biotransformation medium consisted of $20 \mathrm{ml}$ of spore suspension, $30 \mathrm{ml}$ glucose solution $(1 \mathrm{~g} / \mathrm{l})$, and $15 \mathrm{mg}(2.5 \mathrm{mM})$ 2-phenylethanol. Flasks were shaken at $135 \mathrm{rpm}, 24^{\circ} \mathrm{C}$. Biotransformations were carried out from 1 to 14 days (depending on the outcome). After each incubation period, the contents of flasks were centrifuged (15 min, $\left.4{ }^{\circ} \mathrm{C}, 4000 \mathrm{rpm}\right)$, the supernatant was filtered and extracted twice with ethyl acetate $(2 \times 15 \mathrm{ml})$. The organic layers were dried over anhydrous $\mathrm{MgSO}_{4}$ and evaporated under reducing pressure.

\section{Analytical methods}

Cyanobacterial reduction: samples were analyzed using gas chromatography (GC, model 7890A; Agilent
Technologies Co., Ltd.), equipped with a Chiralsil DEX $\mathrm{CB}$ column $25 \mathrm{~m} \times 0.25 \mathrm{~mm}$ and a flame ionization detector with nitrogen as the make-up gas and helium as the carrier gas. The split ratio was $35 / 1$. The column temperature was set at $110^{\circ} \mathrm{C}$, then it was increased to $120^{\circ} \mathrm{C}$ at a rate of $2.5^{\circ} \mathrm{C} / \mathrm{min}$ and subsequently to $200^{\circ} \mathrm{C}$ at a rate of $10^{\circ} \mathrm{C} / \mathrm{min}$. Inlet temperature was $250^{\circ} \mathrm{C}$. Retention times were $4.11 \mathrm{~min}$ for acetophenone, $6.04 \mathrm{~min}$ for $(R)$-1-phenylethanol, and 6.28 min for $(S)$-1-phenylethanol.

The results of $A$. niger biotransformation were evaluated using a high-performance liquid chromatography (Beckman System Gold 126 Solvent Module) with Supelcosil LC-18 column (5 $\mu \mathrm{m}$ particle size, $\mathrm{L} \times$ I.D. $25 \mathrm{~cm} \times 4.6 \mathrm{~mm})$. Acidic $\mathrm{H}_{2} \mathrm{O}(0.1 \%$ formic acid $)$ and acetonitrile were used as the mobile phase at a flow rate of $1 \mathrm{ml} / \mathrm{min}$. The percentage of eluent composition (\% acidic $\mathrm{H}_{2} \mathrm{O} / \%$ acetonitrile) for tyrosol and hydroxytyrosol was 95/5 and for 2-phenylethanol 85/15. The parameters of the absorption and the retention times were different for substrate and products. 2-phenylethanol was analyzed at $\lambda=215 \mathrm{~nm}$, whereas products tyrosol and hydroxytyrosol at $\lambda=276 \mathrm{~nm}$. Compounds were identified by comparing retention times patterns (Brouk and Fishman, 2009).

The spores of Aspergillus niger from sporulated Petri dishes used for biotransformation were counted using flow cytometer (BD FACSVerse).

\section{Results and discussion}

\section{Acetophenone reduction}

The research has proved the ability of cyanobacteria to effectively biotransform acetophenone, prochiral ketone, into corresponding chiral alcohol with high yields and good enantioselectivity (Fig. 1).

The application of cyanobacteria as biocatalysts is not a common approach due to the difficulties in culturing them. Since the presence of other organisms in a culture can interfere with the results, it is also crucial to maintain axenic cyanobacterial cultures. This requirement was confirmed in our research on an agar-solidified BG-11 medium.

Screening experiments were performed for different strains of biocatalysts - filamentous Nodularia sphaerocarpa, Arthrospira maxima, Leptolygbya foveolarum, Geitlerinema sp., unicellular Synechococcus bigranulatus, and heterocystous Nostoc cf-muscorum. 

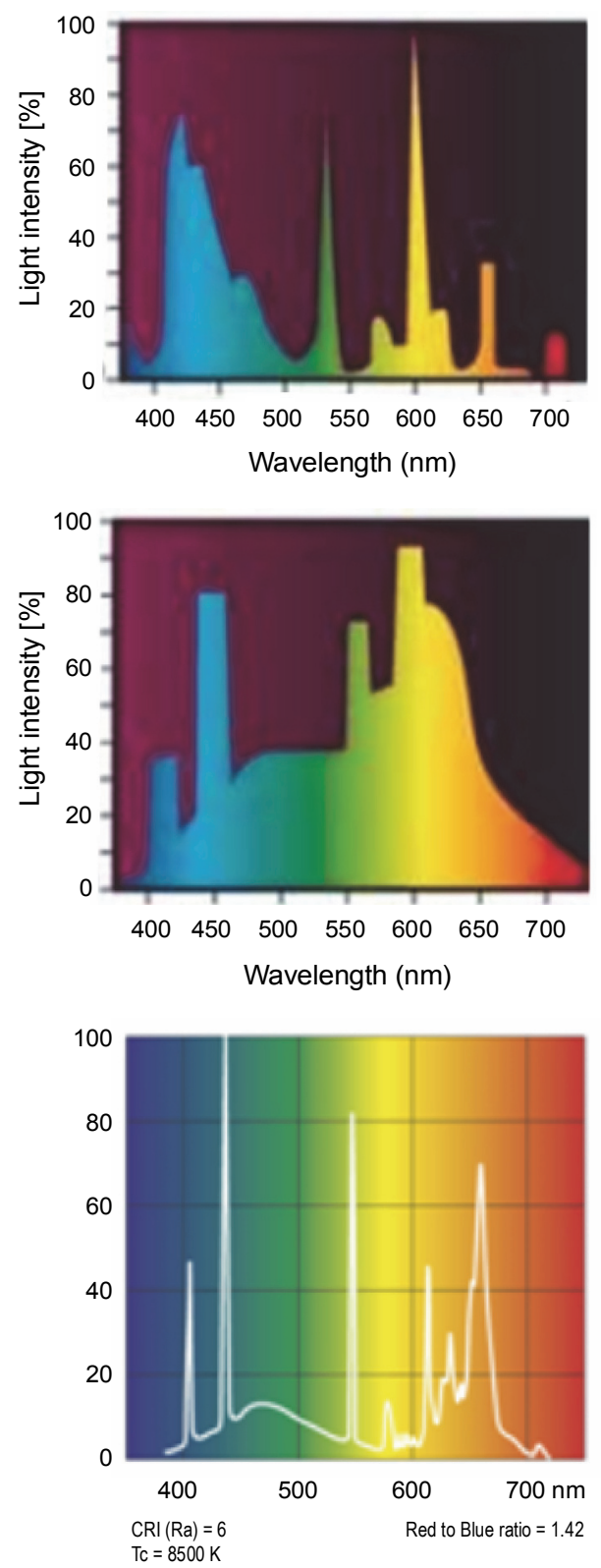

Fig. 3. Light spectra of PowerGlo, SunGlo and Sylvania lamps (provided by manufacturers)

During bacterial cultivation different light sources were used. A PowerGlo fluorescent lamp, which provided bluish white light, SunGlo fluorescent lamp, which imitated daylight, and Sylvania fluorescent lamp, with high proportion of blue and red light in the spectra (Fig. 3). Light regime is one of the most crucial factors which affect the growth of phototrophic microorganisms. The quality of light is essential for their photosynthetic activity, which, in turn, determines the level of every metabolic path and enzymatic activities (including dehydrogenases). There is some evidence that the reduction of exogenously added ketones by cyanobacterial

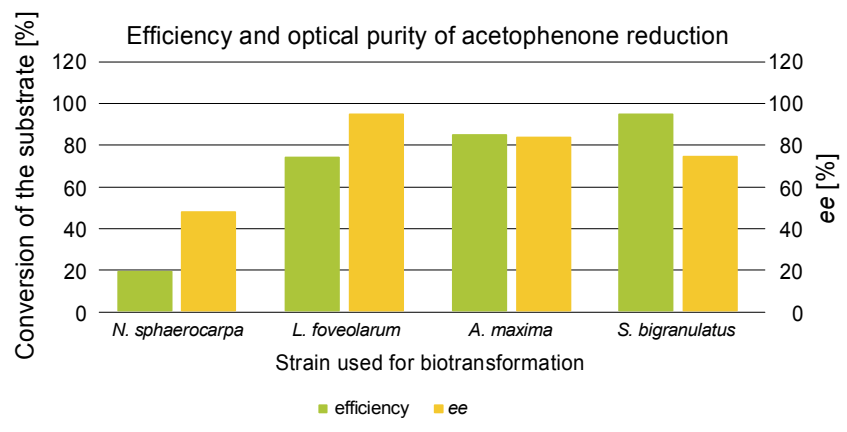

Fig. 4. Summary of acetophenone reduction - efficiency of the reaction and its optical purity

cultures is dependent on light intensity and therefore on the photosynthetic activity of particular strains (Nakamura and Yamanaka, 2002a, 2002b). Cyanobacterial enzymes involved in the reductive processes, also xenobiotics conversion (acetophenone), require reduced forms of coenzymes - mostly nicotinamide adenine dinucleotide phosphate (NADPH), which is one of the products of photosynthetic reactions (Yamanaka et al., 2011). Thus the light regime should be suitable for a process.

Following the general biotransformation procedure using cyanobacteria as biocatalysts (see section Materials and methods, Acetophenone reduction), it turned out that four of them were capable of conducting the reaction of acetophenone reduction. The best conversion degree was obtained by using Nodularia sphaerocarpa $(79.6 \%)$, but the enantioselectivity expressed as enantiomeric excess (ee) was not satisfactory (48\%) and also large amounts of by-products were generated. This microorganism inhabits various ecosystems; therefore, it may have developed mechanisms allowing to survive under conditions of nutrient deficiency. Reaction catalyzed by Leptolyngbya foveolarum resulted in $25.7 \%$ of conversion degree of the substrate with the highest enantiomeric purity (ee 95\%), forming almost exclusively (S)-1-phenylethanol. Using Arthrospira maxima allowed for obtaining a reduced product with $14.6 \%$ of yield and $84 \%$ ee. The last cyanobacterial strain able to perform this reaction was Synechococcus bigranulatus, with $(S)$-phenylethyl alcohol at $4.7 \%$ of the yield and $75 \%$ $e e$. Every tested strain preferably formed ( $S$ )-1-phenylethanol over ( $R$ )-1-phenyethanol (Table 1, Fig. 4), which confirms previous studies concerning the biotransformation of acetophenone derivatives by Synechococcus sp. PCC 7942 into corresponding (S)-alcohols (Nakamura et al., 2000) and asymmetric synthesis of chiral ketones 

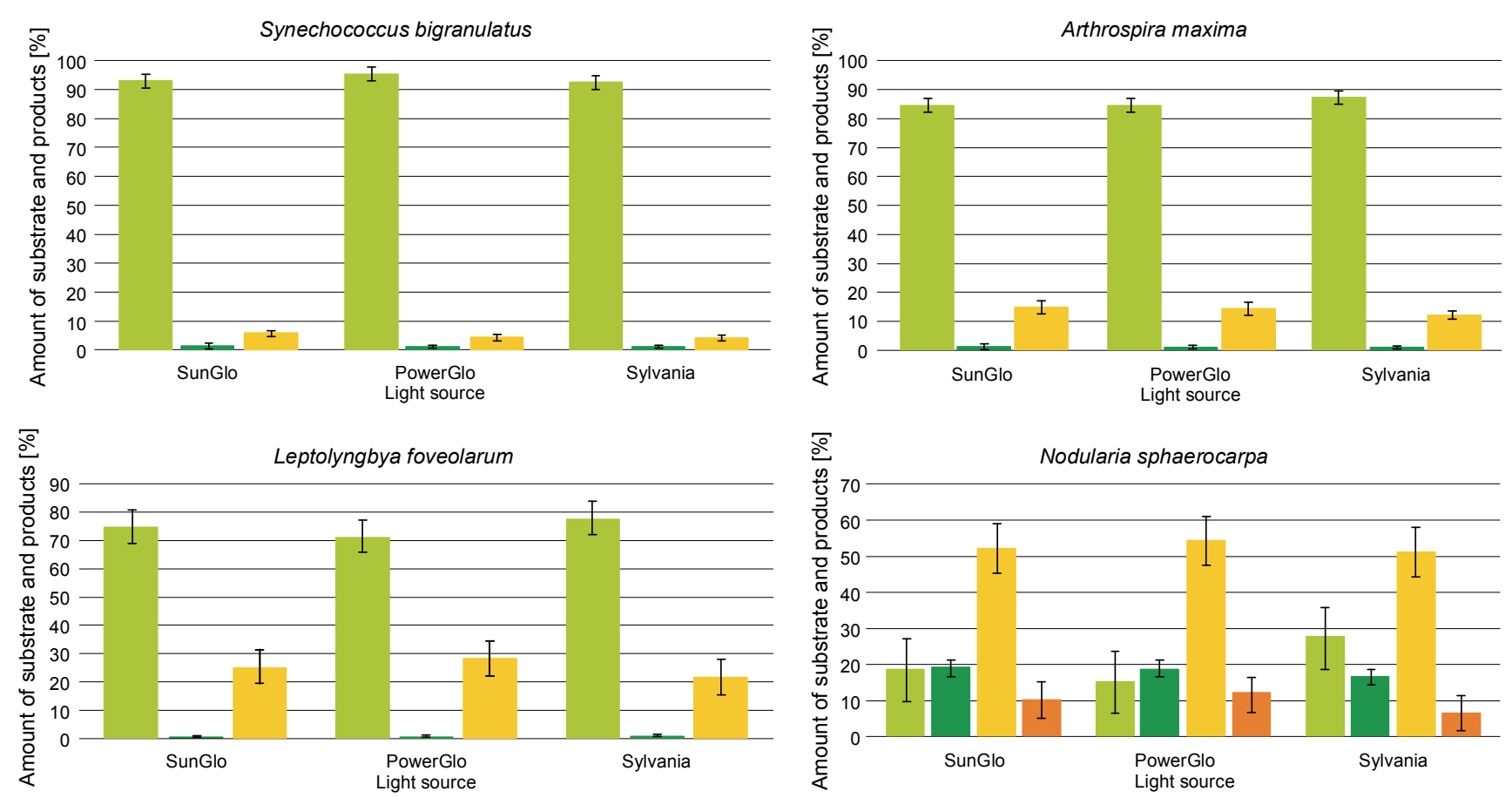

eptolyngbya foveolarum

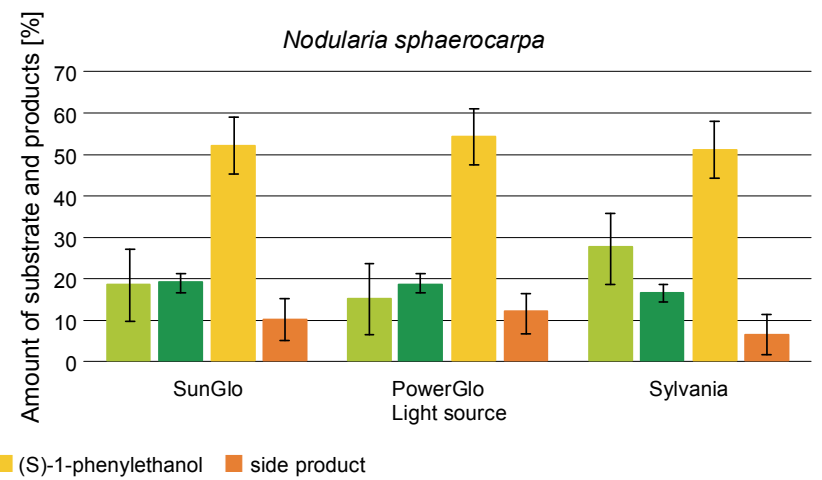

Fig. 5. Bar charts illustrating the efficiency of acetophenone reduction reaction under different lamp regime

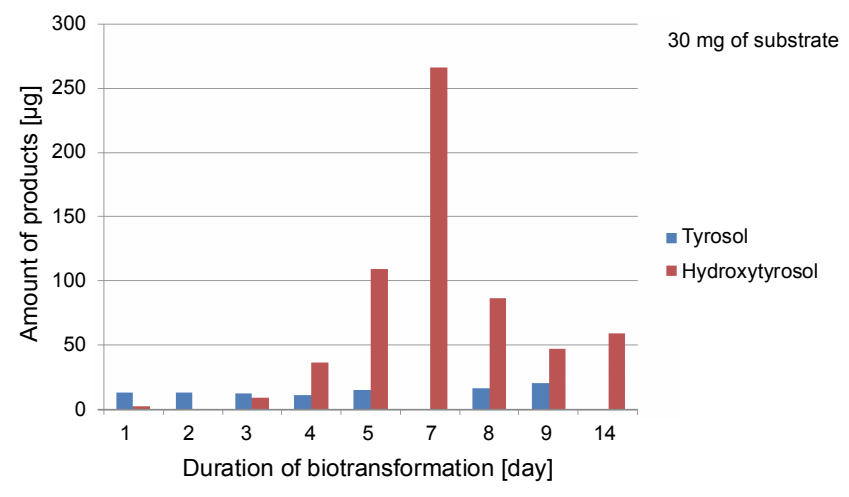

Fig. 6. Results of biotransformation with $30 \mathrm{mg}(8 \mathrm{mM})$ of substrate

from enones performed by the same cyanobacterial strain (Shimoda et al., 2004). The 3-ketoacyl-(acyl-carrier-protein) reductase (EC 1.1.1.100), an enzyme which can be responsible for catalyzing this reaction, has been earlier isolated and characterized (Hölsch et al., 2008). It was also proven that homologous oxidoreductases derived from different strains of cyanobacteria also possess similar enantioselectivity, which may result from the structure of their catalytic tetrad (Hölsch and WeusterBotz, 2010).

The effect of different light sources on the biocatalyzed reactions was also studied. The results have sho-

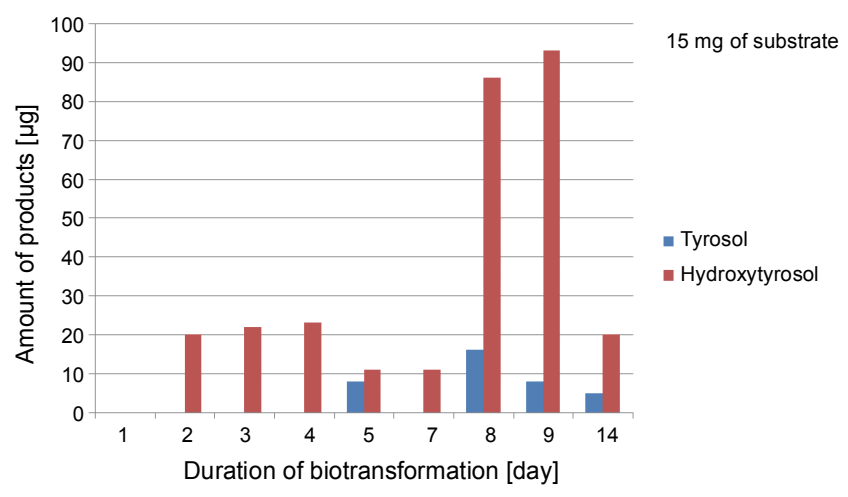

Fig. 7. Results of biotransformation with $15 \mathrm{mg}(4 \mathrm{mM})$ of substrate

wed that the differences in reaction efficiency under different lamps are very small and within the range of statistical error, therefore, it may be concluded that the reaction yield and enantioselectivity are independent of the illumination regime (Fig. 5). This is because cyanobacteria contain mainly phycobiliprotein which absorbs blue-green, green, orange, and yellow light in the range 500-650 nm (Richmond, 2004), and although the light spectra for every lamp are different, they all emit light within this range. Similar results were obtained in the research of biocatalytic potential of cyanobacteria towards phosphonates (Górak and Żymańczyk-Duda, 2015). 


\section{2-Phenylethanol hydroxylation}

Preliminary results show that biohydroxylation of 2-phenylethanol catalyzed by Aspergillus niger leads to the formation of tyrosol and hydroxytyrosol. The experiments performed differed in the substrate concentrations and the duration of biotransformation. A change in the concentration of the starting alcohol may increase or decrease the amount of products formed. A high concentration of the substrate can inhibit the growth of microorganisms and their enzymatic activities or be toxic and result in the induction of stress response mechanisms. The toxicity of some polyphenols can be destructive to DNA and/or RNA, lipids, and proteins. These damages affect crucial biological processes including cellular transport, energy generation, DNA and protein synthesis, and metabolic activities (Nicolaou et al., 2010, Zingaro et al., 2013). It is the reason why there is a need to control the product formation at every step of bioconversion to avoid any toxic effects due to the substrate or products. Also in such context the proper concentration of the applied chemicals is essential for the effectiveness of the process. In the case discussed, better results were achieved for lower concentrations of the starting material, during longer periods of biotransformations. Results of the experiments performed are shown in the graphs (Fig. 6-8).

The initial experiments were done with the use of $8 \mathrm{mM}$ 2-phenylethanol. As the results show, the amount of hydroxytyrosol increased after 7 days of biotransformation but in the following days it decreased (Fig. 6). This may be a result of low hydroxytyrosol stability under the process conditions and its partial splitting (LópezGarcía et al., 2010) or the formation of by-products.

To improve the effectiveness of biohydroxylation, different amounts of substrate were added to the reaction media while maintaining the amount of catalyst at the same level. Experiments with lower concentrations of starting alcohol $(4 \mathrm{mM})$ were performed and the results showed a slightly better effectiveness of the biohydroxylation process (Fig. 7).

However, an increase in the concentration of 2-phenylethanol (up to $17 \mathrm{mM}$ ) resulted in product formation with the worst effectiveness (Fig. 8). A higher concentration of the substrate can inhibit the germination of spores by knocking out the enzymes involved in this process, which may lead to the death of cells (Nicolaou et al., 2010; Zingaro et al., 2013).

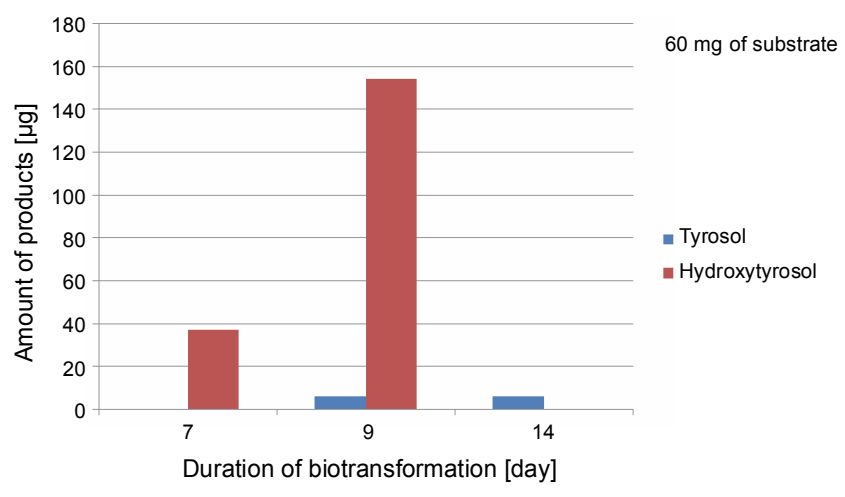

Fig. 8. Results of biotransformation with $60 \mathrm{mg}(17 \mathrm{mM})$ of substrate

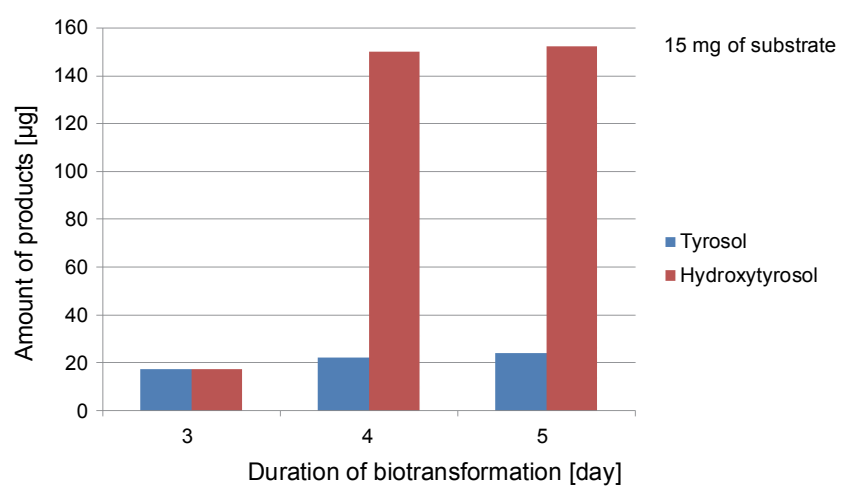

Fig. 9. Results of biotransformation with glucose addition

Because of the known effect of temperature on the metabolisms of viable cells, the effect of this factor on the process was examined. A temporary storage of the spores at a high or low temperature is a commonly applied treatment influencing their germination. This affects the activities of some enzymes and can consequently be used for the improvement of the bioconversions results (Schlegel, 2008). Therefore, in our research, spores were kept in the cold temperature $\left(6^{\circ} \mathrm{C}\right.$ and $-18^{\circ} \mathrm{C}$ ) for two and a half weeks to force the change in the activity of their enzymatic system. However, this approach failed to ensure better effectiveness of the hydroxylation performed.

To increase the enzymatic activity of the fungus, glucose was added to the biotransformation medium. About $30 \mathrm{ml}$ of glucose $(1 \mathrm{~g} / \mathrm{l})$ and $15 \mathrm{mg}(2.5 \mathrm{mM})$ of 2-phenylethanol were added to $20 \mathrm{ml}$ of spore suspension and biotransformation was carried for 3-5 days. The obtained results showed a more effective hydroxylation. After four days of biotransformation of the substrate $(2.5 \mathrm{mM})$, more efficient formation of hydroxytyrosol 
Table 1. Efficiency and optical purity (ee) of the products of acetophenone reduction

\begin{tabular}{l|c|c|c|c|c}
\hline \multirow{2}{*}{ Biocatalyst } & \multirow{2}{*}{$\begin{array}{c}\text { Substrate } \\
\text { acetophenone }\end{array}$} & \multicolumn{3}{|c|}{ Products } & \multirow{2}{*}{ ee } \\
\cline { 3 - 5 } & & $(R)$-1-phenylethanol & $(S)-1$-phenylethanol & Side products & \\
\hline N. sphaerocarpa & $20.4 \%$ & $18.2 \%$ & $52.1 \%$ & $9.4 \%$ & $48 \%$ \\
\hline L. foveolarum & $74.3 \%$ & $0.6 \%$ & $25.1 \%$ & - & $95 \%$ \\
\hline A. maxima & $85.4 \%$ & $1.2 \%$ & $13.4 \%$ & - & $84 \%$ \\
\hline S. bigranulatus & $95.3 \%$ & $0.6 \%$ & $4.2 \%$ & - & $75 \%$ \\
\hline
\end{tabular}

was observed (Fig. 9). Glucose added as a carbon source to the biotransformation medium may act as a part of the coenzyme regenerating system. This can improve the germination of spores, and may be the initiation factor of the first step of the bioconversion ( $\mathrm{Li}$ et al., 2002; Woodyer et al., 2008).

\section{Conclusions}

The research conducted allowed to identify different strains of cyanobacteria active towards acetophenone. The best bioconversion degree, but at the same time low enantioselectivity of the reduction reaction, was achieved with the use of filamentous Nodularia sphaerocarpa. The application of the remaining strains: Arthrospira maxima, unicellular Synechococcus bigranulatus, and mat-forming Leptolyngbya foveolarum gave lower yields, with enantioselectivity reaching 95\% (Table 1). In the case of biohydroxylation of the aromatic ring, the ability of Aspergillus niger to hydroxylate 2-phenylethanol to antioxidants, tyrosol and hydroxytyrosol, has been confirmed. Further studies are intended to optimize the biocatalytic protocol and to increase the efficiency and the scale of the process via modifications of the growing conditions of microorganisms and the biotransformation protocols.

The important finding in this study is that phenolic compounds obtained in biotransformation reactions described earlier could be further used in medicine, chemistry, and cosmetic and food industries.

\section{References}

Abed R.M.M., Dobretsov S., Sudesh K. (2009) Applications of cyanobacteria in biotechnology. J. Appl. Microbiol. 106: $1-12$.

Aiba S., Ogawa T. (1977) Assessment of growth yield of a bluegreen alga Spirulina platensis in axenic and continuous culture. J. Gen. Microbiol. 102: 179-182.
Andreassi M., Andreassi L. (2004) Antioxidants in dermocosmetology: from the laboratory to clinical application. J. Cosmet. Dermatol. 2: 153-160.

Brouk M., Fishman A. (2009) Protein engineering of toluene monooxygenases for synthesis of hydroxytyrosol. Food Chem. 116: 114-121.

Cao Y., Zhuang Y., Yao C., Wu B., He B. (2012) Purification and characterization of an organic solvent-stable lipase from Pseudomonas stutzeri LC2-8 and its application for efficient resolution of $(R, S)-1$-phenylethanol. Biochem. Eng. J. 64: 55-60.

Demyttenaere J., Pooter H. (1998) Biotransformation of citral and nerol by spores of Penicillium digitatum. Flavour Fragr. J. 13: 173-176.

Esmaeili A., Rohany S., Safaiyan S., Zarei S. (2011) Microbial transformation of citral by Aspergillus niger-PTCC 5011 and study of the pathways involved. Czech J. Food Sci. 29 (6): 610-615.

Faber K. (2000) Biotransformations in organic chemistry. Springer Verlag.

Franco M.N., Galeano-Díaz T., López O. (2014) Phenolic compounds and antioxidant capacity of virgin olive oil. Food Chem. 163: 289-298.

Górak M., Żymańczyk-Duda E. (2015) Application of cyanobacteria for chiral phosphonate synthesis. Green Chem. 17: 4570-4578.

Havel J., Wuester-Botz D. (2006) Comparative study of cyanobacteria as biocatalysts for the asymmetric synthesis of chiral building blocks. Eng. Life Sci. 6: 175-179.

Hölsch K., Havel J, Haslbeck M., Weuster-Botz D. (2008) Identification, cloning, and characterization of a novel ketoreductase from the cyanobacterium Synechococcus sp. strain PCC 7942. Appl. Environ. Microbiol. 74 (21): 6697-6702.

Hölsch K., Weuster-Botz D. (2010) New oxidoreductases from cyanobacteria: exploring nature's diversity. Enzyme Microb. Technol. 47: 228-235.

Hu T., He X.W., Jiang J.G., Xu X.L. (2014) Hydroxytyrosol and its potential therapeutic effects. J. Agric. Food Chem. 62: 1449-1455.

Kisukuri C.M., Andrade L.H. (2015) Production of chiral compounds using immobilized cells as a source of biocatalysts. Org. Biomol. Chem. 13: 10086-10107.

Li Z., Beilen J., Duetz W., Schmid A., Raadt A., Griengl H., Witholt B. (2002) Oxidative biotransformations using oxygenases. Curr. Opin. Chem. Biol. 6: 136-144. 
López-García A., López O., Maya I., Fernández-Bolaños J. (2010) Complexation of hydroxytyrosol with b-cyclodex trins. An efficient photoprotection. Tetrahedron 66: 80068011.

Muhr E., Leicht O., Gonzalez Sierra S., Thanbichler M., Heider J. (2016) A fluorescent bioreporter for acetophenone and 1-phenyloethnanol derived from a specifically induced catabolic operon. Frontier. Microbiol. 6: 1561.

Nakamura K., Yamanaka R. (2002a) Light mediated cofactor recycling system in biocatalytic asymmetric reduction of ketone. Chem. Commun. 16: 1782-1783.

Nakamura K., Yamanaka R. (2002b) Light-mediated regulation of asymmetric reduction of ketones by a cyanobacterium. Tetrahedron-Asymmetr. 13(23): 2529-2533.

Nakamura K., Yamanaka R., Tohi K., Hamada H. (2000) Cyanobacterium-catalyzed asymmetric reduction of ketones. Tetrahedron Lett. 41: 6799-6802.

Nicolaou S., Gaida S., Papoutsakis E. (2010)A comparative view of metabolite and substrate stress and tolerance in microbial bioprocessing: from biofuels and chemicals, to biocatalysis and bioremediation. Metabol. Engin. 12: 307-331.

Richmond A. (2004) Handbook of microalgal culture: biotechnology and applied phycology. Blackwell Science.

Rippka R., Deruelles J., Waterbury J.B., Herdman M., Stainer R.Y. (1979) Generic assignments, strain histories and properties of pure cultures of cyanobacteria. J. Gen. Microbiol. 111: 1-61.

Schlegel H. (2008) Allgemeine Mikrobiologie. Thieme.

Schrader J., Etschmann M., Sell D., Hilmer J., Rabenhorst J. (2004) Applied biocatalysis for the synthesis of natural flavour compounds - current industrial processes and future prospects. Biotechnol. Lett. 26: 463-472.

Shimoda K., Kubota N., Hamada H., Kajib M., Hirata T. (2004) Asymmetric reduction of enones with Synechococcus sp. PCC 7942. Tetrahedron-Asymmetr. 15: 1677-1679.

Stark D., Munch T., Sonnleitner B., Marison I., Stockar U. (2002) Extractive bioconversion of 2-phenylethanol from L-phenylalanine by Saccharomyces cerevisiae. Biotechnol. Prog. 18: 514-523

Umeno A., Takashima M., Murotomi K., Nakajima Y., Koike T., Matsuo T., Yoshida Y. (2015) Radical-scavenging activity and antioxidative effects of olive leaf components oleuropein and hydroxytyrosol in comparison with homovanillic alcohol. J. Oleo. Sci. 64(7): 793-800.

Wolken W., Tramper J. (2003) What can spores do for us? Trends Biotechnol. 21(8): 338-345.

Woodyer R., Johannes T., Zhao H. (2008) Regeneration of cofactors for enzyme biocatalysis. [in:] Enzyme technology. Ed. Pandey A., Webb C., Soccol C., Larroche C., New Delhi, Springer: 83-101.

Yamanaka R., Nakamura K., Murakami A. (2011) Reduction of exogenous ketones depends upon NADPH generated photosynthetically in cells of the cyanobacterium Synechococcus PCC 7942. AMB Express. 1: 24.

Zingaro K., Nicolaou S., Papoutsakis E. (2013) Dissecting the assays to assess microbial tolerance to toxic chemicals in bioprocessing. Trends Biotechnol. 31(11): 643-653. 